\title{
The Follow-Up of Thyroid Disease in 188 Pregnant Women Ac- cording to the Guidelines of ATA (American Thyroid Associa- tion) 2017
}

\author{
Sema Yuksekdag ${ }^{1}$, Aysun Firat ${ }^{2}$, Abdullah Yildiz ${ }^{1}$ and Ethem Unal ${ }^{*}$ \\ ${ }^{1}$ Department of General Surgery, Umraniye Education and Research Hospital, Health Sciences University, \\ Istanbul, Turkey \\ ${ }^{2}$ Department of Obstetrics and Gynecology, Istanbul Education and Research Hospital, Health Sciences \\ University, Istanbul, Turkey
}

*Corresponding author: Ethem Unal, MD, Associate Professor, General Surgery and Surgical Oncology, Department of General Surgery, Umraniye Training and Research Hospital, Health Sciences University, Istanbul, Turkey, Tel: +90-532258-4917, Fax: +90-216-632-1818

\begin{abstract}
Background: Thyroid disease in pregnancy is a common clinical problem. Since the guidelines for the management of these disorders by the American Thyroid Association (ATA) were first published in 2011, significant clinical and scientific advances have occurred in the field. A major and substantial change in the new guidelines includes raising the upper limit in the normal thyroid function tests. For Thyroid Stimulating Hormone (TSH), the upper limit was 2.5 $\mathrm{mU} / \mathrm{L}$ in the 2011 guidelines. Normal upper limit in pregnancy is raised to $4.0 \mathrm{mU} / \mathrm{L}$ in 2017 revision. In the present study, we evaluated our pregnant patients according to the ATA 2017 criteria.

Methods: All pregnant women previously followed-up by Internal Medicine, Endocrinology and General Surgery policlinics in one-year period from January 2018 to December 2018 were included in the study. The study was approved by our institution's Ethics Committee (23.01.2019/B.10.1.TK H.4.34.H.GP.0.01/6). Regularly determined thyroid function tests (free T3: Triiodothyronine, free T4: Thyroxine, TPO $\mathrm{Ab}$ : Thyroperoxidase antibody, and TSH: Thyroid stimulating hormon) in all trimesters, medications started in case of necessity and the outcome of pregnancy were documented after a detailed computerized statistical HIS (Health Information System) research.
\end{abstract}

Results: A total of 188 pregnant women (with newly- diagnosed thyroid disease-Group $1 ; \mathrm{n}=23,12.2 \%$, and with previously-known thyroid disease-Group 2, $n=165,87.7 \%$ ) were included. The normal ranges of thyroid tests for non- pregnant women set by our biochemistry laboratory were as follows: T3 (1.71-3.71 pg/mL), T4 (0.7-1.48 ng/dL), TSH (0.35-4.94 ulU/mL) and anti TPO (<5.60 IU/mL). The pregnant women were evaluated according to the ATA guidelines published in 2017, and the normal upper limit in pregnancy was raised to $4.0 \mathrm{ulU} / \mathrm{mL}$ in our institution. The most common thyroid disorders according to ATA 2017 criteria were hypothyroidism ( $n=20,86.9 \%$ for Group 1 and $n=$ $16,9.6 \%$ for Group 2 ) and hyperthyroidism ( $n=3,13 \%$ for Group 1 and $n=4,2.4 \%$ for Group 2). Most of the patients in Group 2 were seen to be euthyroid from the beginning of the pregnancy $(n=145,87.8 \%)$, and they had no remarkable hormone change. Levothyroxine was started in Group 1 (for both clinic and subclinical hypothyroidism, regardless of anti TPO levels), and its dose was adjusted in Group 2 hypothyroidic patients. Propylthiouracil (PTU) was started or methymazol to PTU switch was done in hyperthyroid patients, respectively. There was no significant morbidity during pregnancy and in postpartum period.

Conclusions: Recent studies in Asia, finding only a modest reduction in the upper reference limit of TSH for the typical patient in early pregnancy, with a gradual return to nonpregnant levels later have changed TSH set point as $4 \mathrm{mU} / \mathrm{L}$. Although there might be a relative decrease in the number of pregnant women with thyroid disease regarding the new guidelines, close follow-up of patients and the use and/or adjustments in medications are still important, especially in iodine-deficient areas.

\section{Keywords}

Pregnancy, Hyperthyroidism, Hypothyroidism, ATA, 2017

Citation: Yuksekdag S, Firat A, Yildiz A, Unal E (2020) The Follow-Up of Thyroid Disease in 188 Pregnant Women According to the Guidelines of ATA (American Thyroid Association) 2017. Reprod Med Int 3:012. doi.org/10.23937/2643-4555/1710012

Accepted: February 01, 2020; Published: February 03, 2020

Copyright: (c) 2020 Yuksekdag S, et al. This is an open-access article distributed under the terms of the Creative Commons Attribution License, which permits unrestricted use, distribution, and reproduction in any medium, provided the original author and source are credited. 


\section{Introduction}

Thyroid diseases are very common in pregnancy, and important to treat for a healthy fetal development. During the first trimester, maternal thyroxine is essential for fetal organogenesis as it supplies hypothalamus, pituitary and the thyroid gland; and it is almost complete by 12 to 14 gestational week [1,2]. Then, at around 12 weeks, the thyroid gland in the fetus will begin to produce its own thyroid hormones. During pregnancy, estrogen and human chorionic gonadotropin (hCG) may cause thyroid hormones to rise and occasionally, may cause symptoms similar to hyperthyroidism such as palpitations, weight loss or persistent vomiting [3].

If there is a pre-existing hyperthyroidism or hypothyroidism, one should be more cautious and be sure that medications are properly adjusted, if necessary. It is well-known that untreated thyroid diseases during pregnancy may lead to premature birth, preeclampsia, miscarriage, low birth weight and mental retardation [4]. Since the guidelines for the management of thyroid disorders by the American Thyroid Association (ATA) were first published in 2011, significant clinical and scientific advances have occurred in the field [5]. A major and substantial change in the new guidelines includes raising the upper limit in the normal thyroid function tests. For thyroid stimulating hormone (TSH), the upper limit was $2.5 \mathrm{mU} / \mathrm{L}$ in the 2011 guidelines [6]. Normal upper limit in pregnancy is raised to $4.0 \mathrm{mU} / \mathrm{L}$ in 2017 revision.

In the current study, we evaluated our pregnant patients according to the revised ATA 2017 criteria, and presented our experince in the guidance of updated adjustments.

\section{Patients and Method}

The present study was undertaken after approval of our institution's Ethics Committee (SBU/23.01.2019/ B.10.1.TKH.4.34.H.GP.0.01/6). All pregnant women (among Caucasian White population in a well-known iodine-deficient Euro-Asian area of the world) previously followed-up by Internal Medicine, Endocrinology and General Surgery outpatient clinics in one-year period from January 2018 to December 2018 were included in the study.

Thyroid function tests (free T3: Triiodothyronine, free T4: Thyroxine, TPO Ab: Thyroperoxidase antibody, and TSH, by Architect c4100, Abbott Core Lab Co, Illinois, USA) were requested from all pregnant women regularly (at least once in every 6 weeks if hormone levels are within normal range during follow-up controls or in every 2 or 4 weeks if new medication has been started or the dose is adjusted according to the current hormone level in first trimester or second and third trimesters, respectively).

Follow-up outpatient controls continued through all pregnancy period. Medications started in case of necessity, and the outcome of pregnancy were documented after a detailed computerized statistical HIS (Health Information System) research.

\section{Results}

188 pregnant women (with newly-diagnosed thyroid disease-Group $1 ; n=23,12.2 \%$, and with previously-known thyroid disease-Group $2, n=165,87.7 \%$ ) were included in the study. In our institution, the normal ranges of thyroid tests for non-pregnant women set by our biochemistry laboratory were as follows: free T3 $(1.71-3.71 \mathrm{pg} / \mathrm{mL})$, free T4 $(0.7-1.48 \mathrm{ng} / \mathrm{dL})$, TSH (0.35-4.94 ulU/mL) and anti TPO $(<5.60 \mathrm{lU} / \mathrm{mL})$. The pregnant women were evaluated according to the ATA guidelines published in 2017, and the normal upper limit in pregnancy was raised to $4.0 \mathrm{ulU} /$ $\mathrm{mL}$ and set as normal limit in our clinics.

The most common thyroid disorders according to ATA 2017 criteria were hypothyroidism ( $n=20,86.9 \%$ for Group 1 and $n=16,9.6 \%$ for Group 2) and hyperthyroidism ( $n=3,13 \%$ for Group 1 and $n=4,2.4 \%$ for Group 2). Most of the patients in Group 2 were seen to be euthyroid from the beginning of the pregnancy ( $\mathrm{n}$ $=145,87.8 \%$ ), and they had no remarkable hormone change.

It was seen that $22.8 \%$ (almost $1 / 5$ ) of pregnant women were needed a closer follow-up (at least once per every 4 weeks) as they started to have new medications or dose-changes.

Levothyroxine was started in Group 1 (for both clinic and subclinic hypothyroidism, regardless of TPO Ab levels), and its dose was adjusted in Group 2 hypothyroidic patients. Propylthiouracil (PTU) was started (Group 1) or methimazole to PTU switch was done (Group 2) in all patients with hyperthyroidism, respectively. There was no significant morbidity during pregnancy and in postpartum period.

\section{Discussion}

The most common cause of hypothyroidism in the pregnancy is autoimmune disorders such as Hashimoto's thyroiditis $[2,7]$. It is treated with a synthetic hormone (levothyroxine), and the dose is adjusted and monitored by thyroid function tests every 4-6 weeks during pregnancy (ATA recommendation) $[5,6]$. In our clinic, we follow-up our patients every 2 weeks during the first trimester and every 4 weeks after then if the diagnosis is new or hormone levels fluctuates unreliably. During the first trimester of pregnancy, fetal brain development is totally dependent on the maternal thyroid hormones [8]. Since congenital hypothyroidism is one of the most preventable causes of mental retardation, we insist on the necessity of frequent blood tests especially valid for the first 14 weeks. It is also vital to remember that iron and calci- 
um in prenatal vitamins may block the absorption of thyroid medications, one should not take these vitamins within 4 hours of taking levothyroxine [9].

The most common cause of maternal hyperthyroidism during pregnancy is Graves' disease [10]. An antibody called thyroid-stimulating immunoglobulin (TSI) levels increase and causes the thyroid to overreact. TSI may cause developing fetus to begin to produce more hormone than it needs. Therefore, an anti-thyroid medication that blocks production of thyroid hormones is necessary. As we use in our own practice in pregnant women with hyperthyroidism, propylthiouracil (PTU) is started or methimazole (teratogenic) to PTU switch is done in those patients hyperthyroid patients in the first trimester.

Since many recent studies (mostly from Asia) have suggested that the fixed upper limits for TSH of 2.5 or $3.0 \mathrm{mU} / \mathrm{L}$ are too low and might lead to overdiagnosis or even overtreatment, new guidelines of the ATA (2017) set this cut-off level to $4 \mathrm{mU} / \mathrm{L}$ in pregnancy [11]. 2017 ATA guidelines now recommend the following: 1) Calculate pregnancy-specific and lab-specific reference ranges for TSH and free $\mathrm{T} 4 ; 2$ ) If it is not possible, adopt a reference range from the literature that is derived using a similar assay and preferably also in a population with similar characteristics (ethnicity, BMI, iodine status etc.); and 3) If 1 and 2 are not possible, deduct $0.5 \mathrm{mU} / \mathrm{I}$ from the nonpregnancy reference range (which in most centers would results in a cut-off of roughly $4.0 \mathrm{mU} / \mathrm{l}$ ) [6]. The results of our study approves the expediency of 2017 recommendations, since we have no remarkable morbidity.

In conclusion, when the new ATA criterias for pregnant women with thyroid disorders are taken into consideration, one can presume a relative decrease in the number of pregnant women necessitating closer follow-ups. However, in our opinion, close follow-up and the use and/or adjustments of medications are still important in pregnant women with thyroid disordes, especially in the iodine-deficient areas of the world.

\section{Conflict of Interest}

None.

\section{Financial Support}

None.

Ethics' Committe-approved

(SBU/23.01.2019/

B.10.1.TKH.4.34.H.GP.0.01/6).

\section{References}

1. Springer D, Jiskra J, Limanova Z, Zima T, Potlukova E (2017) Thyroid in pregnancy: From physiology to screening. Crit Rev Clin Lab Sci 54: 102-116.

2. Sahay RK, Nagesh VS (2012) Hypothyroidism in pregnancy. Indian J Endocrinol Metab 16: 364-370.

3. Alemu A, Terefe B, Abebe M, Biadgo B (2016) Thyroid hormone dysfunction during pregnancy: A review. Int J Reprod Biomed 14: 677-686

4. Tingi E, Syed AA, Kyriacou A, Mastorakos G, Kyriacou A (2016) Benign thyroid disease in pregnancy: A state of the art review. J Clin Transl Endocrinol 6: 37-49.

5. Stagnaro-Green A, Abalovich M, Alexander E, Azizi F, Mestman JE, et al. (2011) Guidelines of the American Thyroid Association for the diagnosis and management of thyroid disease during pregnancy and postpartum. Thyroid 21: 1081-1125.

6. Alexander EK, Pearce EN, Brent GA, Brown RS, Chen $\mathrm{H}$, et al. (2017) 2017 Guidelines of the American Thyroid Association for the Diagnosis and Management of Thyroid Disease During Pregnancy and the Postpartum. Thyroid 27: 315-389.

7. Rotondi M, Chiovato L, Pacini F, Bartalena L, Vitti P (2018) Management of subclinical hypothyroidism in pregnancy: $A$ comment from the Italian Society of Endocrinology and the Italian Thyroid Association to the 2017 American Thyroid Association guidelines-“The Italian way". Thyroid 28: 551555.

8. Jansen TA, Korevaar TIM, Mulder TA, White T, Muetzel RI, et al. (2019) Maternal thyroid function during pregnancy and child brain morphology: a time window-specific analysis of a prospective cohort. Lancet Diabetes Endocrinol 7: 629-637.

9. Kashi Z, Bahar A, Akha O, Hassanzade S, Esmaeilisaraji L, et al. (2015) Levothyroxine dosage requirement during pregnancy in well-controlled hypothyroid women: A longitudinal study. Glob J Health Sci 8: 227-233.

10. Kobaly K, Mandel SJ (2019) Hyperthyroidism and Pregnancy. Endocrinol Metab Clin North Am 48: 533-545.

11. Korevaar TIM (2018) The upper limit for TSH during pregnancy: why we should stop using fixed limits of 2.5 or 3.0 mU/l. Thyroid Res 11: 5. 